\title{
Construction of intelligent fire management system based on BIM technology
}

\section{Linqing HUANG ${ }^{1,}$, , Yongxu XU ${ }^{2, b}$, Xiaofeng $\operatorname{LIAO}^{3, c}$, Lingling $\mathrm{QIN}^{4, \mathrm{~d}}$ and Jiangting QIU $5, \mathrm{e}$}

${ }^{1}$ School of Civil Engineering and Architecture, Chongqing University of Science \& Technology, No. 20, East Road, University City, Shapingba District, Chongqing

${ }^{2}$ School of Civil Engineering and Architecture, Chongqing University of Science \& Technology, No. 20, East Road, University City, Shapingba District, Chongqing

${ }^{3}$ School of Civil Engineering and Architecture, Chongqing University of Science \& Technology, No. 20, East Road, University City, Shapingba District, Chongqing

${ }^{4}$ School of Civil Engineering and Architecture, Chongqing University of Science \& Technology, No. 20, East Road, University City, Shapingba District, Chongqing

${ }^{5}$ School of Civil Engineering and Architecture, Chongqing University of Science \& Technology, No. 20, East Road, University City, Shapingba District, Chongqing

ahlinqing@126.com, b1147429454@qq.com, c10895315@qq.com, d2586612090@qq.com, eg48172136@qq.com

Keywords: BIM technology; intelligent fire control monitoring system; information sharing; rescue and escape route

Abstract: At present, the casualties and property losses caused by urban building fire are on the rise in China. Based on BIM technology, this paper analyzes the feasibility about building the indoor real-time intelligent linkage fire management system, besides demonstrates it from the technical level, and puts forward the construction idea of monitoring system. The construction of the system can make up for the lack of command and rescue from the fire department, and can realize the joint rescue of the fire department and the Property union command, as well as the rescue of the fire victims' self-rescue.

\section{Preface}

In recent years, along with the acceleration of urbanization in our country, the construction, expansion and reconstruction projects are springing up, and the residence is developing towards large capacity, large span, high-rise and super high-rise. The complex structure and huge space in these buildings greatly increase the difficulty of building fire. However, when fire happens, no matter the location and the trend of late development of fire, all of them have strong randomness. So how to determine fire location quickly and accurately and effectively evacuate personnel in building are being very important. Nevertheless the evacuation in the building is mainly a two-dimensional evacuation map. Although it plays a certain role, it still has some defects such as poor visibility ${ }^{[1]}$. And which cannot adjust the escape route ${ }^{[2-3]}$ according to the specific circumstances of the fire. At the same time, because of the complexity of building structure, the two-dimensional model of evacuation plan will be difficult to show effectively the relationship among spatial information, equipment information, personnel information, and it is very difficult to find fire protection equipment and location safety exit for people who are not familiar with the building, and under the harsh environment such as the smoke interference, rescue workers are difficult to familiar quickly with the construction drawings and find out the fire line. Besides internal staff of construction are also very difficult to quickly find the evacuation route of escape, which is extremely unfavorable to the ${ }^{[4]}$ evacuation route choice, meanwhile different fire point directly affect the evacuation route choice. Therefore, the traditional way to evacuate the sketch map as an evacuation guide is unable to meet the evacuation guide demand of large buildings ${ }^{[5]}$. In addition, the location and fire size of the fire are 
very random, so the traditional and static evacuation guide is very difficult to carry out efficient evacuation guide ${ }^{[6-7]}$.

In view of the traditional fire management system can't fully understand the scene around the fire, fire area layout and structure information, and then which can't help firefighters to find the location and the cause of the fire and select the fire extinguishing and rescue path in the shortest time, this paper presents an intelligent fire management system of 3D visualization. On the basis of BIM technology, the 3D information model, which includes the information of all components, the overall structure information of the project and indoor fire control facilities and other information, is established. And then use of internet of things to achieve the real-time updating of model information, which will greatly improve the communication efficiency of the traditional graphic drawings and the rescue efficiency.

\section{Construction of intelligent fire monitoring and management system based on BIM technology}

\section{Module design of intelligent fire monitoring and management system}

The intelligent fire monitoring and management system consists of six modules: information collection module, model module, intelligent processing module, decision module, information feedback module and escape terminal APP module.

(1)Information collection module

In order to realize the real-time collection and update of the information and ensure the accuracy of the information, the existing internet of things equipment such as monitoring, smoke feeling, fire control sign and so on are used to collect the field information and efficiently understand the situation on the spot.

(2)Model module

The BIM model of the building is established by using BIM technology. The model includes the information of all the components in the building, the information of water, electricity and gas and all the information of fire extinguishing equipment, thus which can provide data support for indoor escape and rescue of building firefighting.

(3)Intelligent processing module

The intelligent processing module can automatically plan the rescue path, rescue tools, indoor escape path, and modify the contents of fire protection sign by synthetically processing the data of the information collection module and the model module.

(4)Decision module

The rescue center reads the information on the equipment and models of internet of things, and other information through the decision module, and issues the emergency evacuation command, at the same time, which chooses the outdoor rescue routes and rescue tools, and sends rescue orders to the rescue teams.

(5)Information feedback module

After the decision is made, the information feedback module automatically releases the best escape route to the survivors, and indicates the location of the fire rescue tools nearby, besides provides the best rescue route, rescue ways, rescue tools and other information to the rescuers, and relieves the alarm in time after the rescuing.

(6)Escape terminal APP module

Once fire occurred, the information center promptly pushed the escape route to the user's mobile phone and issued the alarm on the mobile phone. The survivors can check the location of the fire through the mobile phone terminal APP. The intelligent processing module will push the best escape path and nearby rescue tools into the APP and highlight them for the survivors to choose.

\section{Realization of intelligent fire monitoring and management system}

The realization of intelligent fire monitoring management system is mainly through the integration of information, the combination of internet of things technology and the construction of cloud platform, as shown in Figure 1 below. 


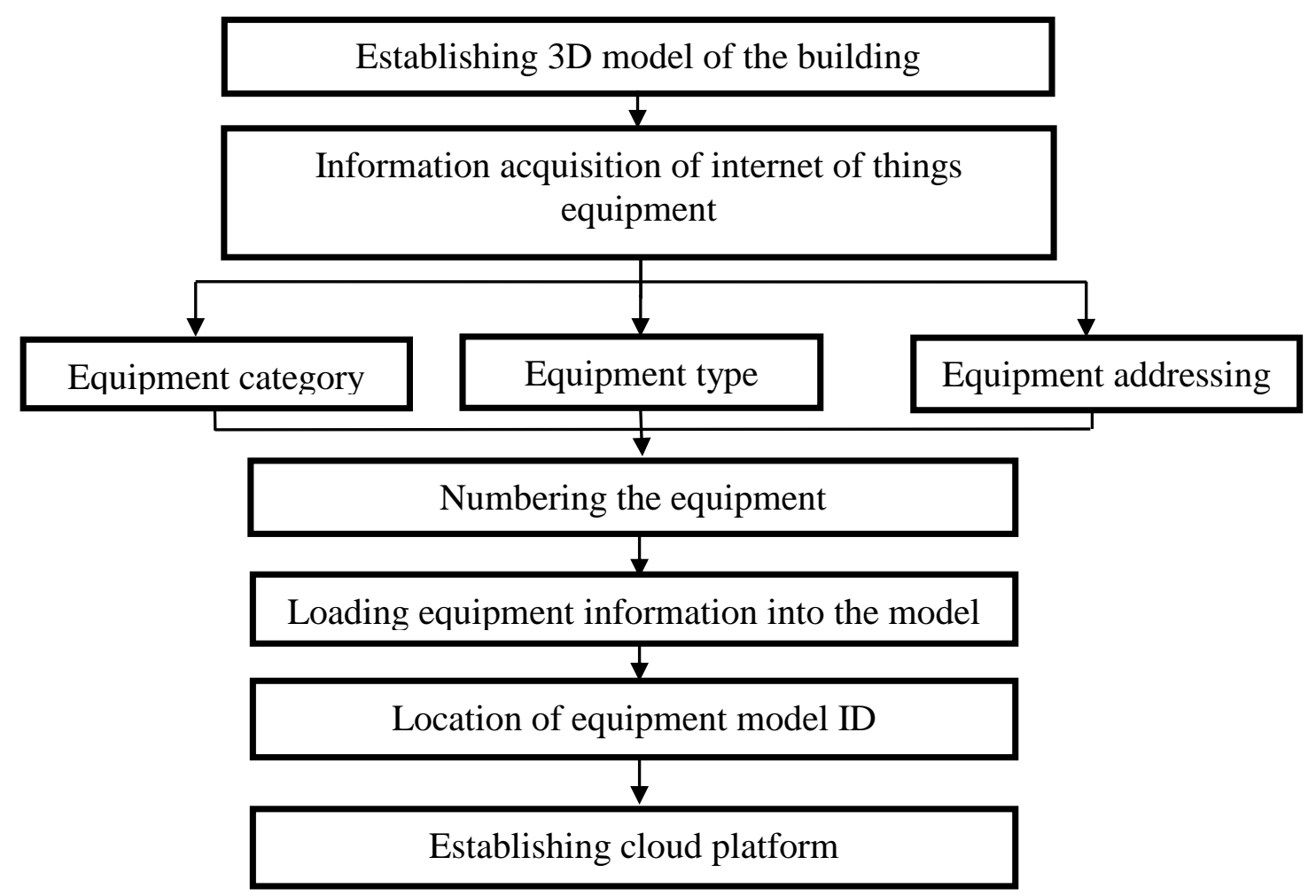

(1)BIM information integration

Figure 1 system realization way

Using BIM technology establish the 3D buildings model, the model can integrate the whole life cycle data of the building into the BIM model, such as building structure, spatial layout, each stage of participant data and other information and so on, which includes the water pipe position, gas position, power lines and other tenants of the building, besides we can screen and call any information about construction at any time, and the information is true and only, furthermore these data are normalization and propitious to data exchange, which can content the intelligent fire monitoring and management system to planning the rescue routes, selecting tool and other function for the actual information needs.

(2)BIM model integrated internet of things equipment

BIM model integrated with the internet of things equipment, in fact, it is to connect the existing internet of things equipment with the model, through the BIM model, we can achieve these functions, which include monitoring equipment information, modifying the fire indicator display content, locating rescue tools and other functions. First of all, the smoke sense, monitoring equipment, firefighting equipment and other internet of things equipment type and location information are collected. If it is the transformation of the old building, at first we should set up a three-dimensional model, and then the model collects the installed internet of things equipment information, after that number the equipment, and last according to the actual location of the equipment corresponding into the model, at the same time marking the model location ID information. The type and number information of the equipment is also indicated for the modified location. For the new project, the BIM model is directly used here, and the information is loaded into the model after the installation of the equipment.

(3)Instant messaging technology based on cloud platform

Through the cloud technology, among the rescue center, rescuers, survivors and on-site equipment data information can achieve real-time interaction, and rescue center through the cloud platform announce the rescue, emergency evacuation and other orders. The rescuers receive the rescue orders through the cloud platform, as well as select the rescue tools and route according to the system prompts, view and understand the scene and the surrounding environment, and the survivors choose the best escape path through the cloud platform. And through the APP to check the three-dimensional 
escape path and related escape tips. Through cloud technology, it not only greatly reduces the time of information transmission but also improves the efficiency of rescue.

\section{Work flow of intelligent fire control system}

The work flow of the system is shown in Figure 2 below.

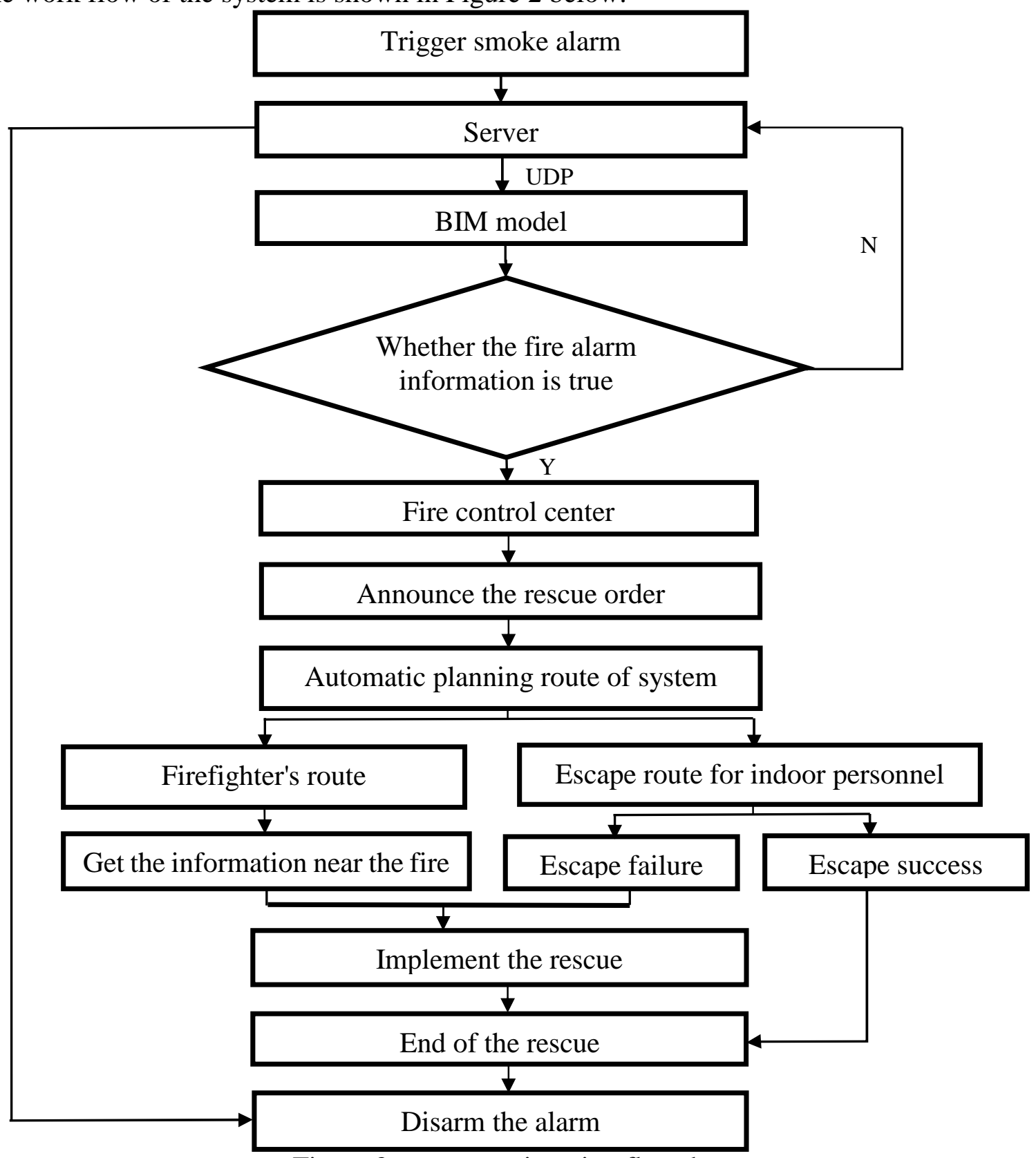

Figure 2 system engineering flowchart

With the help of existing sensor and server technology, when smoke sensor alarm occurs, at the same time, the alarm information is passed to the server, and the server collects the incoming information. The server transmits the collected information to the BIM model by the User Datagram Protocol (UDP), in which the alarm location is highlighted. Meanwhile, all firefighting equipment information and the whole floor information are quickly sent out. The server will analyze and record the data transmitted by the sensor. When alarm information is proved to be true. The system automatically alarm through the connection with the fire center, at the same time issue alarm, and warn the indoor personnel to evacuate quickly. Within the evacuation process, survivors access to escape path through the mobile phone APP rapid. The path planning determines the safe route according to the first-out position of the survivor, the information pushed by the received sensor and the firefighting equipment nearby. In the case of greater fire, the survivors are given a relatively safe position. To the fire rescue route, at first we should ensure the location to be rescued, and then obtain 
the floor information, including the secondary hazard sources such as gas pipeline and power line of the floor, finally plan a rescue route. We should bypass the alarm when survivors are rescued. If a false alarm is detected within the data processing phase, the system will push the code directly to the server. After the server output command, the alarm is bypassed.

\section{Conclusions}

The intelligent fire management system is proposed in this paper, which makes full use of the fire information, realizes the information sharing of all parties, and improves the rescue ability of trapped persons and rescuers when the fire occurs. However, information collection, centralized processing and how to connect the information with the model to ensure the effectiveness of information and other factors, have a great impact on the overall practicality and reliability of the system, and is also the guarantee of maximizing the success of self-rescue and rescue. Therefore, strengthening the management of fire information will greatly improve the power of fire rescue, and it is of great significance to improve fire safety.

\section{Acknowledgements}

This work was financially supported by the Chongqing land resources and housing administration research project $(\mathrm{KJ} 2017007)$

\section{References}

[1] Xiaoyu Bi: Research on the generation and optimization of building fire emergency plan (Beijing Architecture University, China 2014).

[2] Yongheng Zhao, Dengjun Tong: Application of intelligent fire emergency lighting evacuation indicator system (The spread of science and technology magazine, China 2014).

[3] Lianyi Zhang, Hao Sun, Laihao Zhao: Analysis of intelligent fire evacuation system (Science and Technology Innovation Herald, China 2017).

[4] Shuhui Li: Construction and development of firefighting information (Fire Technique and Products Information, China 2011).

[5] Xue Gao, Jia Wang, Junyan Yi: Study on the guidance of evacuation route in building based on BIM Technology (Architecture Science, China 2016).

[6] Xu Jiang: Research on the application of intelligent management system for firefighting equipment under the technology of Internet of things (Industry and Application Security, China 2017).

[7] Zhiyong Wang, Guang Li, Bo Gao: Design of intelligent fire control system based on Internet of things (Journal of Hebei Engineering and Technical College Quarterly, China 2017). 